\title{
Fractional calculus and application of generalized Struve function
}

\author{
Kottakkaran Sooppy Nisar ${ }^{1 *}$ (B), Dumitru Baleanu ${ }^{2}$ and Maysaa' Mohamed Al Qurashi ${ }^{3}$
}

\author{
${ }^{*}$ Correspondence: \\ n.sooppy@psau.edu.sa \\ ${ }^{1}$ Department \\ of Mathematics, College \\ of Arts and Science, Prince \\ Sattam Bin Abdulaziz \\ University, 11991 Wadi \\ Aldawser, Saudi Arabia \\ Full list of author information \\ is available at the end of the \\ article
}

\begin{abstract}
A new generalization of Struve function called generalized Galué type Struve function (GTSF) is defined and the integral operators involving Appell's functions, or Horn's function in the kernel is applied on it. The obtained results are expressed in terms of the Fox-Wright function. As an application of newly defined generalized GTSF, we aim at presenting solutions of certain general families of fractional kinetic equations associated with the Galue type generalization of Struve function. The generality of the GTSF will help to find several familiar and novel fractional kinetic equations. The obtained results are general in nature and it is useful to investigate many problems in applied mathematical science.
\end{abstract}

Keywords: Fractional calculus, Generalized Struve function, Integral transforms, Fractional kinetic equations, Laplace transforms

Mathematics Subject Classification: Primary 26A33, 44A20; Secondary 33E12, 44A10

\section{Background}

Fractional calculus has found many demonstrated applications in extensive areas of applied science such as dynamical system in control theory, viscoelasticity, electrochemistry, signal processing and model of neurons in biology (Podlubny 1999; Hilfer 2000; Adjabi et al. 2016; Baleanu et al. 2016; Kilbas et al. 2006; Glöckle and Nonnenmacher 1991; Mathai et al. 2010). Recent studies observed that the solutions of fractional order differential equations could model real-life situations better, particularly in reactiondiffusion type problems. Due to the potential applicability to wide variety of problems, fractional calculus is developed to large area of Mathematics physics and other engineering applications. Several researchers have investigated fractional kinetic equations as its possible applications in diverse physical problems. In this connection, one can refer to the monograph by various works (Saichev and Zaslavsky 1997; Haubold and Mathai 2000; Saxena et al. 2002, 2004, 2006; Saxena and Kalla 2008; Chaurasia and Pandey 2008; Gupta and Sharma 2011; Chouhan and Sarswat 2012; Chouhan et al. 2013; Gupta and Parihar 2014). Recently, many papers investigated the solutions of generalized fractional kinetic equations (GFKE) involving various types of special functions. For instance, the solutions of GFKE involving M-series (Chaurasia and Kumar 2010), generalized Bessel function of the first kind (Kumar et al. 2015), Aleph function (Choi and Kumar 2015)

(c) 2016 The Author(s). This article is distributed under the terms of the Creative Commons Attribution 4.0 International License (http://creativecommons.org/licenses/by/4.0/), which permits unrestricted use, distribution, and reproduction in any medium, provided you give appropriate credit to the original author(s) and the source, provide a link to the Creative Commons license, and indicate if changes were made. 
and the generalized Struve function of the first kind (Nisar et al. 2016b). Here, in this paper, we aim at presenting the integral transforms and the solutions of certain general families of fractional kinetic equations associated with newly defined Galué type generalization of Struve function.

Galué (2003) introduced a generalization of the Bessel function of order $p$ given by

$$
{ }_{a} J_{p}(x):=\sum_{k=0}^{\infty} \frac{(-1)^{k}}{\Gamma(a k+p+1) k !}\left(\frac{x}{2}\right)^{2 k+p}, \quad x \in \mathbb{R}, a \in \mathbb{N}=\{1,2,3, \ldots\}
$$

Baricz (2010) investigated Galué-type generalization of modified Bessel function as:

$$
{ }_{a} I_{p}(x):=\sum_{k=0}^{\infty} \frac{1}{\Gamma(a k+p+1) k !}\left(\frac{x}{2}\right)^{2 k+p}, \quad x \in \mathbb{R}, a \in \mathbb{N}
$$

The Struve function of order $p$ given by

$$
H_{p}(x):=\sum_{k=0}^{\infty} \frac{(-1)^{k}}{\Gamma(k+3 / 2) \Gamma\left(k+p+\frac{3}{2}\right)}\left(\frac{x}{2}\right)^{2 k+p+1},
$$

is a particular solution of the non-homogeneous Bessel differential equation

$$
x^{2} y^{\prime \prime}(x)+x y^{\prime}(x)+\left(x^{2}-p^{2}\right) y(x)=\frac{4\left(\frac{x}{2}\right)^{p+1}}{\sqrt{\pi} \Gamma(p+1 / 2)}
$$

where $\Gamma$ is the classical gamma function whose Euler's integral is given by (see, e.g., Srivastava and Choi 2012, Section 1.1):

$$
\Gamma(z)=\int_{0}^{\infty} e^{-t} t^{z-1} d t, \quad \operatorname{Re}(z)>0
$$

The Struve function and its more generalizations are found in many papers (Bhowmick 1962, 1963; Kanth 1981; Singh 1974; Nisar and Atangana 2016; Singh 1985, 1988a, b, 1989). The generalized Struve function given by Bhowmick (1962)

$$
H_{l}^{\lambda}(x)=\sum_{k=0}^{\infty} \frac{(-1)^{k}\left(\frac{t}{2}\right)^{2 k+l+1}}{\Gamma\left(\lambda k+l+\frac{3}{2}\right) \Gamma\left(k+\frac{3}{2}\right)}, \quad \lambda>0
$$

and by Kanth (1981)

$$
H_{l}^{\lambda, \alpha}(x)=\sum_{k=0}^{\infty} \frac{(-1)^{k}\left(\frac{x}{2}\right)^{2 k+l+1}}{\Gamma\left(\lambda k+l+\frac{3}{2}\right) \Gamma\left(\alpha k+\frac{3}{2}\right)}, \quad \lambda>0, \alpha>0
$$

Singh (1974) found another generalized form as

$$
H_{l, \xi}^{\lambda}(x)=\sum_{k=0}^{\infty} \frac{(-1)^{k}\left(\frac{x}{2}\right)^{2 k+l+1}}{\Gamma\left(\lambda k+\frac{l}{\xi}+\frac{3}{2}\right) \Gamma\left(k+\frac{3}{2}\right)}, \quad \lambda>0, \xi>0
$$


The generalized Struve function of four parameters was given by Singh (1985) (also, see Nisar and Atangana 2016) as:

$$
H_{p, \mu}^{\lambda, \alpha}(x):=\sum_{k=0}^{\infty} \frac{(-1)^{k}}{\Gamma(\alpha k+\mu) \Gamma\left(\lambda k+p+\frac{3}{2}\right)}\left(\frac{x}{2}\right)^{2 k+p+1}, \quad p, \lambda \in \mathbb{C}
$$

where $\lambda>0, \alpha>0$ and $\mu$ is an arbitrary parameter. Another generalization of Struve function by Orhan and Yagmur $(2014,2013)$ is,

$$
\mathcal{H}_{p, b, c}(z):=\sum_{k=0}^{\infty} \frac{(-c)^{k}}{\Gamma(k+3 / 2) \Gamma\left(k+p+\frac{b}{2}+1\right)}\left(\frac{z}{2}\right)^{2 k+p+1}, \quad p, b, c \in \mathbb{C}
$$

Motivated from (1), (3) and (10), here we define the following generalized form of Struve function named as generalized Galué type Struve function (GTSF) as:

$$
{ }_{a} \mathcal{W}_{p, b, c, \xi}^{\alpha, \mu}(z):=\sum_{k=0}^{\infty} \frac{(-c)^{k}}{\Gamma(\alpha k+\mu) \Gamma\left(a k+\frac{p}{\xi}+\frac{b+2}{2}\right)}\left(\frac{z}{2}\right)^{2 k+p+1}, \quad a \in \mathbb{N}, p, b, c \in \mathbb{C}
$$

where $\alpha>0, \xi>0$ and $\mu$ is an arbitrary parameter and studied fractional integral representations of generalized GTSF.

The generalized integral transforms defined for $x>0$ and $\lambda, \sigma, \vartheta \in \mathbb{C}$ with $\mathfrak{R}(\lambda)>0$ are given in Saigo (1977), (also, see Samko et al. 1987) respectively as

$$
\left(I_{0_{+}}^{\lambda, \sigma, \vartheta} f\right)(x)=\frac{x^{-\lambda-\sigma}}{\Gamma(\lambda)} \int_{0}^{x}(x-t)^{\lambda-1}{ }_{2} F_{1}\left(\lambda+\sigma,-\vartheta ; \lambda ; 1-\frac{t}{x}\right) f(t) d t
$$

and

$$
\left(I_{-}^{\lambda, \sigma, \vartheta} f\right)(x)=\frac{1}{\Gamma(\lambda)} \int_{x}^{\infty}(t-x)^{\lambda-1} t^{-\lambda-\sigma}{ }_{2} F_{1}\left(\lambda+\sigma,-\vartheta ; \lambda ; 1-\frac{x}{t}\right) f(t) d t,
$$

where $\Gamma(\lambda)$ is the familiar Gamma function (see, e.g., Srivastava and Choi 2012, Section 1.1) and ${ }_{p} F_{q}$ is the generalized hypergeometric series defined by (see, e.g., Rainville 1960, p. 73):

$$
\begin{aligned}
{ }_{p} F_{q}\left[\begin{array}{l}
\alpha_{1}, \ldots, \alpha_{p} ; \\
\beta_{1}, \ldots, \beta_{q} ; z
\end{array}\right] & =\sum_{n=0}^{\infty} \frac{\left(\alpha_{1}\right)_{n} \cdots\left(\alpha_{p}\right)_{n}}{\left(\beta_{1}\right)_{n} \cdots\left(\beta_{q}\right)_{n}} \frac{z^{n}}{n !} \\
& ={ }_{p} F_{q}\left(\alpha_{1}, \ldots, \alpha_{p} ; \beta_{1}, \ldots, \beta_{q} ; z\right),
\end{aligned}
$$

$(\lambda)_{n}$ being the Pochhammer symbol defined (for $\lambda \in \mathbb{C}$ ) by (see Srivastava and Choi 2012, p. 2 and p. 5):

$$
\begin{aligned}
(\lambda)_{n}: & = \begin{cases}1 & (n=0) \\
\lambda(\lambda+1) \ldots(\lambda+n-1) & (n \in \mathbb{N})\end{cases} \\
& =\frac{\Gamma(\lambda+n)}{\Gamma(\lambda)} \quad\left(\lambda \in \mathbb{C} \backslash \mathbb{Z}_{0}^{-}\right) .
\end{aligned}
$$


The results given in Kiryakova (1977), Miller and Ross (1993), Srivastava et al. (2006) can be referred for some basic results on fractional calculus. The Fox-Wright function ${ }_{p} \Psi_{q}$ defined by (see, for details, Srivastava and Karlsson 1985, p. 21)

$$
\begin{aligned}
{ }_{p} \Psi_{q}[z] & ={ }_{p} \Psi_{q}\left[\begin{array}{c}
\left(a_{1}, \alpha_{1}\right), \ldots,\left(a_{p}, \alpha_{p}\right) ; \\
\left(b_{1}, \beta_{1}\right), \ldots,\left(b_{q}, \beta_{q}\right) ;
\end{array}\right]={ }_{p} \Psi_{q}\left[\begin{array}{c}
\left(a_{i}, \alpha_{i}\right)_{1, p} ; \\
\left(b_{j}, \beta_{j}\right)_{1, q} ;
\end{array}\right] \\
& =\sum_{n=0}^{\infty} \frac{\prod_{i=1}^{p} \Gamma\left(a_{i}+\alpha_{i} n\right)}{\prod_{j=1}^{q} \Gamma\left(b_{j}+\beta_{j} n\right)} \frac{z^{n}}{n !},
\end{aligned}
$$

where the coefficients $\alpha_{1}, \ldots, \alpha_{p}, \beta_{1}, \ldots, \beta_{q} \in \mathbb{R}^{+}$such that

$$
1+\sum_{j=1}^{q} \beta_{j}-\sum_{i=1}^{p} \alpha_{i} \geqq 0
$$

For more detailed properties of ${ }_{p} \Psi_{q}$ including its asymptotic behavior, one may refer to works (for example Kilbas and Sebastian 2008; Kilbas et al. 2002; Kilbas and Sebastian 2010; Srivastava 2007; Wright 1940a, b).

\section{Fractional integration of (11)}

The following lemmas proved in Kilbas and Sebastian (2008) are needed to prove our main results.

Lemma 1 (Kilbas and Sebastian 2008) Let $\lambda, \sigma, \vartheta \in \mathbb{C}$ be $\ni \quad \mathfrak{R}(\lambda)>0$, $\mathfrak{R}(\rho)>\max [0, \mathfrak{R}(\sigma-\vartheta)]$. Then $\exists$ the relation

$$
\left(I_{0_{+}}^{\lambda, \sigma, \vartheta} t^{\rho-1}\right)(x)=\frac{\Gamma(\rho) \Gamma(\rho+\vartheta-\sigma)}{\Gamma(\rho-\sigma) \Gamma(\rho+\lambda+\vartheta)} x^{\rho-\sigma-1}
$$

Lemma 2 (Kilbas and Sebastian 2008) Let $\lambda, \sigma, \vartheta \in \mathbb{C}$ be $\ni \mathfrak{R}(\lambda)>0$, $\mathfrak{R}(\rho)<1+\min [\Re(\sigma), \mathfrak{R}(\vartheta)]$. Then

$$
\left(I_{-}^{\lambda, \sigma, \vartheta} t^{\rho-1}\right)(x)=\frac{\Gamma(\sigma-\rho+1) \Gamma(\vartheta-\rho+1)}{\Gamma(1-\rho) \Gamma(\lambda+\sigma+\vartheta-\rho+1)} x^{\rho-\sigma-1} .
$$

The main results are given in the following theorem.

Theorem 1 Let $a \in \mathbb{N}, \lambda, \sigma, \vartheta, \rho, l, b, c \in \mathbb{C}, \alpha>0$ and $\mu$ is an any arbitrary parameter be such that $\frac{l}{\xi}+\frac{b}{2} \neq-1,-2,-3, \ldots, \mathfrak{R}(\lambda)>0, \mathfrak{R}(\rho+l+1)>\max [0, \mathfrak{R}(\sigma-\vartheta)]$. Then

$$
\begin{aligned}
& \left(I_{0_{+}}^{\lambda, \sigma, \vartheta} t^{\rho-1} a \mathcal{W}_{l, b, c, \xi}^{\alpha, \mu}(t)\right)(x) \\
& =\frac{x^{l+\rho-\sigma}}{2^{l+1}} \\
& \times_{3} \Psi_{4}\left[\begin{array}{cl|c}
(l+\rho+1,2), & (l+1+\rho+\vartheta-\sigma, 2),(1,1) & -\frac{c x^{2}}{4}
\end{array}\right] .
\end{aligned}
$$


Proof Notice that the condition given in Eq. (17) holds for ${ }_{3} \Psi_{4}$ given in (20) and then interchanging the integration and summation, (11) and (12) together imply

$$
\left(I_{0_{+}}^{\lambda, \sigma, \vartheta} t^{\rho-1}{ }_{a} \mathcal{W}_{l, b, c, \xi}^{\alpha, \mu}(t)\right)(x)=\sum_{k=0}^{\infty} \frac{(-c)^{k}(2)^{-(l+2 k+1)}}{\Gamma(\alpha k+\mu) \Gamma\left(a k+\frac{l}{\xi}+\frac{b+2}{2}\right)}\left(I_{0_{+}}^{\lambda, \sigma, \vartheta} t^{l+2 k+\rho}\right)(x) .
$$

For any $k=0,1,2, \ldots$, clearly $\mathfrak{R}(l+2 k+\rho+1) \geq \mathfrak{R}(\rho+l+1)>\max [0, \mathfrak{R}(\sigma-\vartheta)]$ and hence by Lemma 1 ,

$$
\begin{aligned}
& \left(I_{0_{+}}^{\lambda, \sigma, \vartheta} t^{\rho-1}{ }_{a} \mathcal{W}_{l, b, c, \xi}^{\alpha, \mu}(t)\right)(x) \\
& =\frac{x^{l+\rho-\sigma}}{2^{l+1}} \\
& \quad \times \sum_{k=0}^{\infty} \frac{\Gamma(l+1+\rho+2 k) \Gamma(l+1+\rho+\vartheta-\sigma+2 k)\left(\frac{-c x^{2}}{4}\right)^{k}}{\Gamma(\alpha k+\mu) \Gamma\left(a k+\frac{l}{\xi}+\frac{b+2}{2}\right) \Gamma(l+1+\rho-\sigma+2 k) \Gamma(l+1+\rho+\lambda+\vartheta+2 k)}
\end{aligned}
$$

In view of definition of Fox-Wright function (16) we obtain the desired result.

If we set $\alpha=a=1, \mu=\frac{3}{2}$ and $\xi=1$ in Theorem 1 then we obtain the theorem 1 of Nisar et al. (2016a) as follows:

Corollary 1 Let $\lambda, \sigma, l, b, c \in \mathbb{C} \quad$ be $\quad \ni(l+b / 2) \neq-1,-2,-3 \ldots, \quad \mathfrak{R}(\lambda)>0$, $\mathfrak{R}(\rho+l+1)>0$. Then

$$
\begin{aligned}
& \left(I_{0_{+}}^{\lambda, \sigma, \vartheta} t^{\rho-1} \mathcal{H}_{l, b, c}(t)\right)(x) \\
& =\frac{x^{l+1+\rho-\sigma}}{2^{l+1}} \\
& \times_{3} \Psi_{4}\left[\begin{array}{ccc}
(l+1+\rho, 2), & (l+1+\rho+\vartheta-\sigma, 2),(1,1) \\
\left(l+1+\frac{b}{2}, 1\right),(l+1+\rho-\sigma, 2), & (l+1+\rho+\lambda+\vartheta, 2),\left(\frac{3}{2}, 1\right)
\end{array} \mid-\frac{c x^{2}}{4}\right] .
\end{aligned}
$$

where $\mathcal{H}_{l, b, c}(t)$ is given in (10)

Theorem 2 Let $a \in \mathbb{N}, \lambda, \sigma, \vartheta, \rho, l, b, c \in \mathbb{C}, \alpha>0$ and $\mu$ is an any arbitrary parameter be such that $\left(\frac{l}{\xi}+\frac{b}{2}\right) \neq-1,-2,-3 \ldots, \mathfrak{R}(\lambda)>0$, and $\mathfrak{R}(\rho-l)<2+\min [\mathfrak{R}(\rho), \mathfrak{R}(\vartheta)]$. Then

$$
\begin{aligned}
& \left(I_{-}^{\lambda, \sigma, \vartheta} t^{\rho-1}{ }_{a} \mathcal{W}_{l, b, c, \xi}^{\alpha, \mu}\left(\frac{1}{t}\right)\right)(x) \\
& =\frac{x^{\rho-l-\sigma-2}}{2^{l+1}}
\end{aligned}
$$

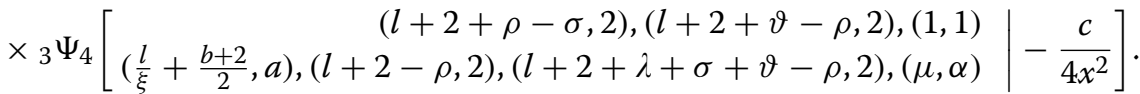


Proof The Fox-Wright function ${ }_{3} \Psi_{4}$ given in (22) is well-defined as it satisfy inequality (17) and changing the order of integration and summation, (13) and (16) together imply

$$
\left(I_{-}^{\lambda, \sigma, \vartheta} t^{\rho-1}{ }_{a} \mathcal{W}_{l, b, c, \xi}^{\alpha, \mu}\left(\frac{1}{t}\right)\right)(x)=\sum_{k=0}^{\infty} \frac{(-c)^{k}(2)^{-(l+2 k+1)}}{\Gamma(\alpha k+\mu) \Gamma\left(a k+\frac{l}{\xi}+\frac{b+2}{2}\right)}\left(I_{-}^{\lambda, \sigma, \vartheta} t^{\rho-l-2-2 k}\right)(x)
$$

Now using Lemma 2 and the under the conditions mentioned in Theorem 2, we have

$$
\begin{aligned}
& \left(I_{-}^{\lambda, \sigma, \vartheta} t^{\rho-1}{ }_{a} \mathcal{W}_{l, b, c, \xi}^{\alpha, \mu}\left(\frac{1}{t}\right)\right)(x) \\
& =\frac{x^{\rho-l-\sigma-2}}{2^{l+1}} \\
& \quad \times \sum_{k=0}^{\infty} \frac{\Gamma(\sigma-\rho+l+2+2 k) \Gamma(\vartheta-\rho+l+2+2 k)}{\Gamma(l+2-\rho+2 k) \Gamma(\lambda+\sigma+\vartheta-\rho+l+2+2 k) \Gamma(\alpha k+\mu) \Gamma\left(a k+\frac{l}{\xi}+\frac{b+2}{2}\right)}\left(-\frac{c}{4 x^{2}}\right)^{k} .
\end{aligned}
$$

Now (22) can be deduced from (23) by using (17), hence the proof.

If we take $\alpha=a=1, \mu=\frac{3}{2}$ and $\xi=1$ in Theorem 2 then we obtain the theorem 2 of Nisar et al. (2016a) as:

Corollary 2 Let $\lambda, \sigma, l, b, c \in \mathbb{C}$ be $\ni(l+b / 2) \neq-1,-2,-3 \ldots, \mathfrak{R}(\lambda)>0$, and $\mathfrak{R}(\rho-l)<2+\min [\mathfrak{R}(\sigma), \mathfrak{R}(\vartheta)]$. Then

$$
\begin{aligned}
& \left(I_{-}^{\lambda, \sigma, \vartheta} t^{\rho-1} \mathcal{H}_{l, b, c}\left(\frac{1}{t}\right)\right)(x) \\
& =\frac{x^{\rho-l-\sigma-2}}{2^{l+1}} \\
& \times{ }_{3} \Psi_{4}\left[\begin{array}{r}
(l+2+\sigma-\rho, 2),(l+2+\vartheta-\rho, 2),(1,1) \\
\left(l+\frac{b+1}{2}, 1\right),(l+2-\rho, 2),(l+2+\lambda+\sigma+\vartheta-\rho, 2),\left(\frac{3}{2}, 1\right)
\end{array} \mid-\frac{c}{4 x^{2}}\right] .
\end{aligned}
$$

where $\mathcal{H}_{l, b, c}(t)$ is given in $(10)$

\section{Application}

In this section, we infer the solution of fractional kinetic equation including generalized GTSF as an application. For this investigation, we need the following definitions:

The Swedish mathematician Mittag-Leffler introduced the so called Mittag-Leffler function $E_{\alpha}(z)$ (see Mittag-Leffler 1905):

$$
E_{\alpha}(z)=\sum_{n=0}^{\infty} \frac{z^{n}}{\Gamma(\alpha n+1)} \quad(z, \alpha \in \mathbb{C} ;|z|<0, \mathcal{R}(\alpha)>0) .
$$

and $E_{\mu, \eta}(z)$ defined by Wiman (1905) as

$$
E_{\mu, \eta}(z)=\sum_{n=0}^{\infty} \frac{z^{n}}{\Gamma(\mu n+\eta)}, \quad(\mu, \eta \in \mathbb{C} ; \mathcal{R}(\mu)>0, \mathcal{R}(\eta)>0) .
$$


The familiar Riemann-Liouville fractional integral operator (see, e.g., Miller and Ross 1993; Kilbas et al. 2006) defined by

$$
{ }_{0} D_{t}^{-v} f(t)=\frac{1}{\Gamma(v)} \int_{0}^{t}(t-s)^{v-1} f(s) d s, \quad \mathcal{R}(v)>0
$$

and the Laplace transform of Riemann-Liouville fractional integral operator ( Erdélyi et al. 1954; Srivastava and Saxena 2001) is

$$
L\left\{{ }_{0} D_{t}^{-v} f(t) ; p\right\}=p^{-v} F(p)
$$

where $F(p)$ is the Laplace transform of $f(t)$ is given by

$$
\begin{aligned}
F(p) & =\mathcal{L}\{f(t): p\}=\int_{0}^{\infty} e^{-p t} f(t) d t \\
& =\lim _{\tau \rightarrow \infty} \int_{0}^{\tau} e^{-p t} f(t) d t
\end{aligned}
$$

whenever the limit exist (as a finite number).

\section{Kinetic equations}

The standard kinetic equation is of the form,

$$
\frac{d N_{i}}{d t}=-c_{i} N_{i}(t)
$$

with $N_{i}(t=0)=N_{0}$, which is the number of density of species $i$ at time $t=0$ and $c_{i}>0$. The integration of (29) gives an alternate form as follows:

$$
N(t)-N_{0}=-c \cdot{ }_{0} D_{t}^{-1} N(t)
$$

where ${ }_{0} D_{t}^{-1}$ is the special case of the Riemann-Liouville integral operator and c is a constant. The fractional generalization of (30) is given by Haubold and Mathai (2000) as:

$$
N(t)-N_{0}=-c_{0}^{v} D_{t}^{-v} N(t)
$$

where ${ }_{0} D_{t}^{-v}$ defined in (26).

Recently, Saxena and Kalla (2008) considered the following equation

$$
N(t)-N_{0} f(t)=-c^{v} \cdot D_{t}^{-v} N(t), \quad \operatorname{Re}(v)>0, c>0
$$

and obtained the solution as:

$$
N(t)=N_{0} \sum_{k=0}^{\infty}(-1)^{k} \frac{c^{k v}}{\Gamma(k v)} t^{k v-1} * f(t)
$$

where

$$
t^{k v-1} * f(t)=\int_{0}^{t}(t-u)^{k v-1} f(u) d u .
$$


For more details about the solution of kinetic equations interesting readers can refer (Saxena and Kalla 2008; Nisar and Atangana 2016).

\section{Solution of fractional kinetic equation involving (11)}

In this section, we will discuss about the solution fractional kinetic equation involving newly defined function generalized GTSF to show the potential of newly defined function in application level.

Given the equation

$$
N(t)-N_{0} a \mathcal{W}_{l, b, c, \xi}^{\alpha, \mu}(t):=-e_{0}^{v} D_{t}^{-v} N(t)
$$

where $e, t, v \in R^{+}, a, b, c, l \in \mathbb{C}$ and $\mathcal{R}(l)>-1$.

Taking the Laplace transform of (34) and using (11) and (27), gives

$$
\begin{aligned}
\mathcal{N}(p)= & N_{0}\left(\int_{0}^{\infty} e^{-p t} \sum_{k=0}^{\infty} \frac{(-c)^{k}}{\Gamma(\alpha k+\mu) \Gamma\left(a k+\frac{l}{\xi}+\frac{b+2}{2}\right)}\left(\frac{t}{2}\right)^{2 k+l+1}\right) d t \\
& -e^{v} p^{-v} \mathcal{N}(p)
\end{aligned}
$$

where $\mathcal{N}(p)=L\{N(t) ; p\}$

Integrate the integral in (35) term by term which guaranteed under the given restrictions and using (5), we get: for $\operatorname{Re}(p)>0$

$$
\left(1+\left(\frac{e}{p}\right)^{\nu}\right) \mathcal{N}(p)=N_{0} \sum_{k=0}^{\infty} \frac{(-c)^{k} 2^{-(2 k+l+1)}}{\Gamma(\alpha k+\mu) \Gamma\left(a k+\frac{l}{\xi}+\frac{b+2}{2}\right)} \frac{\Gamma(2 k+l+2)}{p^{2 k+l+2}}
$$

Taking the geometric series expansion of $\left(1+\left(\frac{e}{p}\right)^{v}\right)^{-1}$, we have: for $e<|p|$

$$
\begin{aligned}
\mathcal{N}(p)= & N_{0} \sum_{k=0}^{\infty} \frac{(-c)^{k}(2)^{-(2 k+l+1)} \Gamma(2 k+l+2)}{\Gamma(\alpha k+\mu) \Gamma\left(a k+\frac{l}{\xi}+\frac{b+2}{2}\right) p^{(2 k+l+2)}} \\
& \times \sum_{r=0}^{\infty}(-1)^{r}\left(\frac{e}{p}\right)^{v r}
\end{aligned}
$$

Applying the inverse Laplace transform and using the following known formula:

$$
L^{-1}\left[p^{-v}\right]=\frac{t^{v-1}}{\Gamma(v)}, \quad \mathcal{R}(v)>0
$$

we have

$$
\begin{aligned}
N(t)= & L^{-1}\{\mathcal{N}(p)\} \\
= & N_{0} \sum_{k=0}^{\infty} \frac{(-c)^{k} \Gamma(2 k+l+2)}{\Gamma(\alpha k+\mu) \Gamma\left(a k+\frac{l}{\xi}+\frac{b+2}{2}\right)}\left(\frac{t}{2}\right)^{2 k+l+1} \\
& \times\left\{\sum_{r=0}^{\infty} \frac{(-1)^{r}(e t)^{v r}}{\Gamma(v r+l+2 k+2)}\right\}
\end{aligned}
$$

In view of Eq. (25), we get, 


$$
N(t)=N_{0} \sum_{k=0}^{\infty} \frac{(-c)^{k} \Gamma(2 k+l+2)}{\Gamma(\alpha k+\mu) \Gamma\left(a k+\frac{l}{\xi}+\frac{b+2}{2}\right)}\left(\frac{t}{2}\right)^{2 k+l+1} E_{v, 2 k+l+2}\left(-e^{v} t^{v}\right) .
$$

The following results are more general than (38) and they can derive parallel as above, so the details are omitted.

Let $e, t, v \in R^{+}, a, b, c, l \in \mathbb{C}$ with $\mathcal{R}(l)>-1$ then the equation

$$
N(t)-N_{0} a \mathcal{W}_{p, b, c, \xi}^{\alpha, \mu}\left(e^{v} t^{v}\right)=-e^{v}{ }_{0} D_{t}^{-v} N(t)
$$

have the following solution

$$
N(t)=N_{0} \sum_{k=0}^{\infty} \frac{(-c)^{k} \Gamma(2 k v+v l+v+1)}{\Gamma(\alpha k+\mu) \Gamma\left(a k+\frac{l}{\xi}+\frac{b+2}{2}\right)}\left(\frac{t^{v} e^{v}}{2}\right)^{2 k+l+1} E_{v,(2 k+l+1) v+1}\left(-e^{v} t^{v}\right)
$$

and the solution of the equation

$$
N(t)-N_{0 a} \mathcal{W}_{p, b, c, \xi}^{\alpha, \mu}\left(e^{v} t^{v}\right)=-\mathfrak{a}_{0}^{v} D_{t}^{-v} N(t)
$$

is

$$
\begin{aligned}
N(t)= & N_{0} \sum_{k=0}^{\infty} \frac{(-c)^{k} \Gamma(2 k v+v l+v+1)}{\Gamma(\alpha k+\mu) \Gamma\left(a k+\frac{l}{\xi}+\frac{b+2}{2}\right)}\left(\frac{e^{v}}{2}\right)^{2 k+l+1} \\
& \times t^{v(2 k+l+1)} E_{v,(2 k+l+1) v+1}\left(-\mathfrak{a}^{v} t^{v}\right)
\end{aligned}
$$

where $\mathfrak{a} \neq e$. The Figs. 1, 2, 3, 4, 5 and 6 are presented to show the behavior of the solution $N(t)$ for different values of $a$ and $v$. The comparison between solutions of GFKE involving generalized Bessel function (solid green line) and generalized Galué type generalization of Struve function (dashed red line) are shown in Fig. 7.

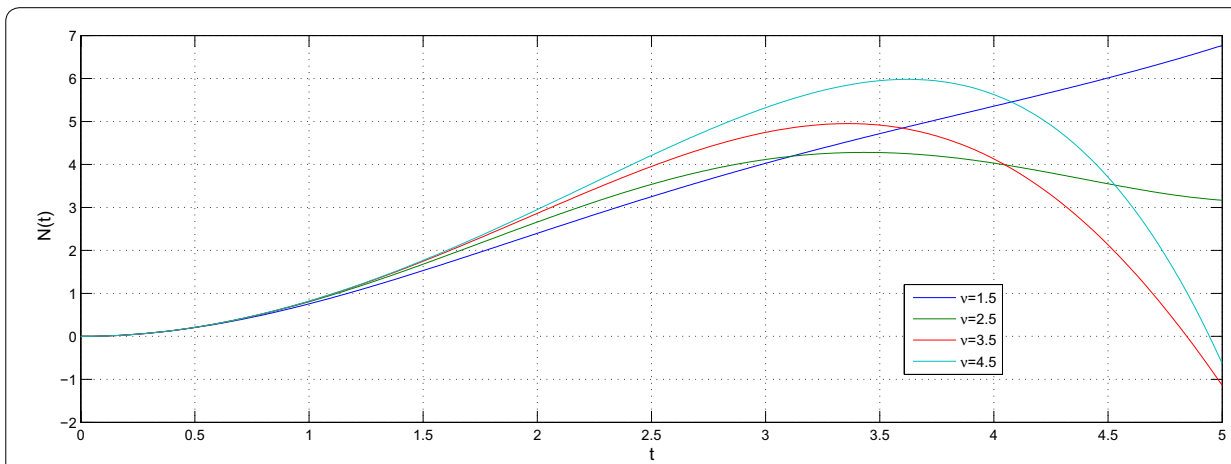

Fig. 1 Solution (38) for $a=1, N_{0}=1, \alpha=\mu=\xi=1$ and $b=c=l=e=1$ 


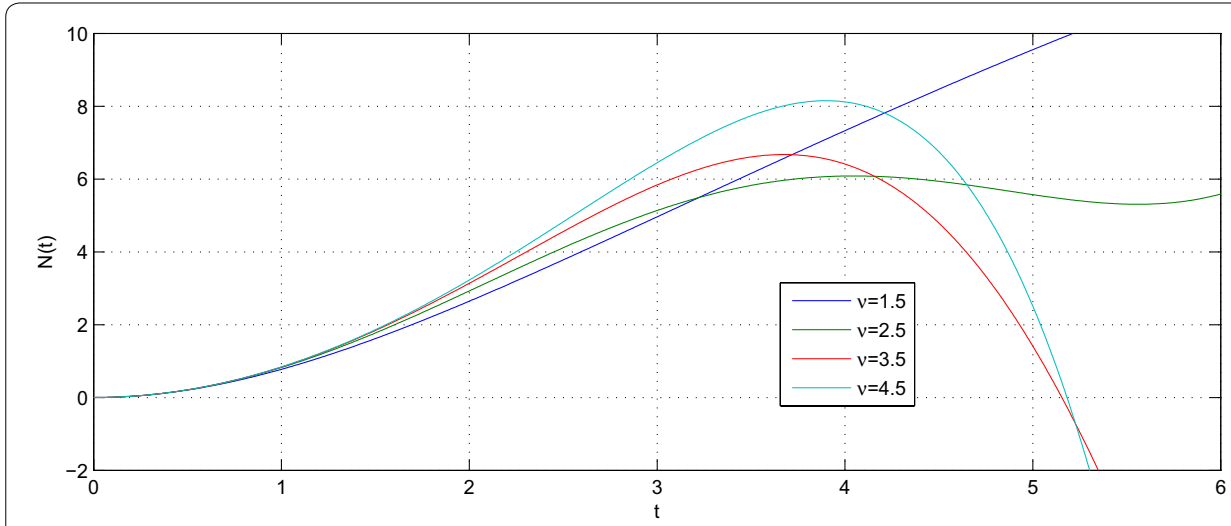

Fig. 2 Solution (38) for $a=2, N_{0}=1, \alpha=\mu=\xi=1$ and $b=c=l=e=1$

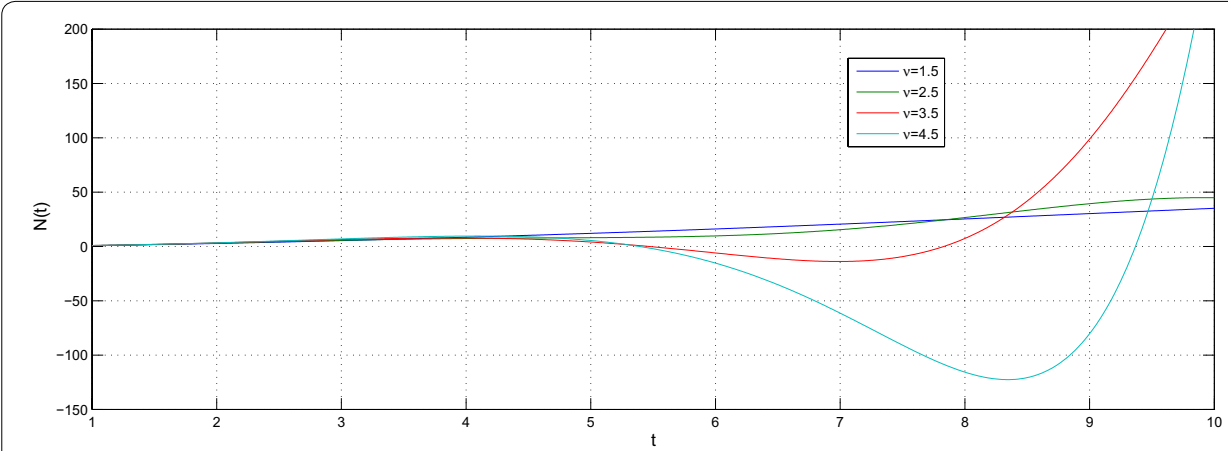

Fig. 3 Solution (38) for $a=3, N_{0}=1, \alpha=\mu=\xi=1$ and $b=c=l=e=1$

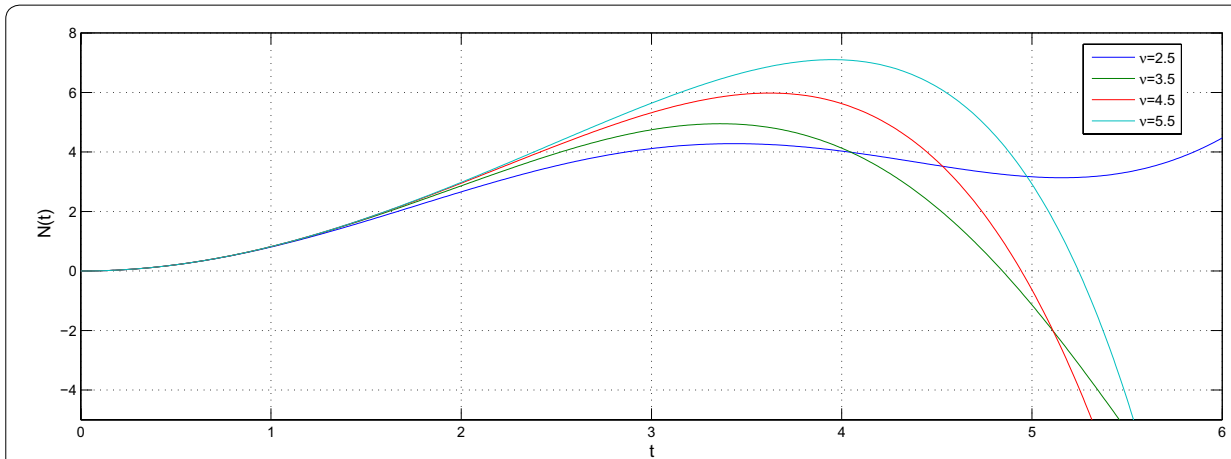

Fig. 4 Solution (40) for $a=1, N_{0}=1, \alpha=\mu=\xi=1$ and $b=c=l=e=1$ 


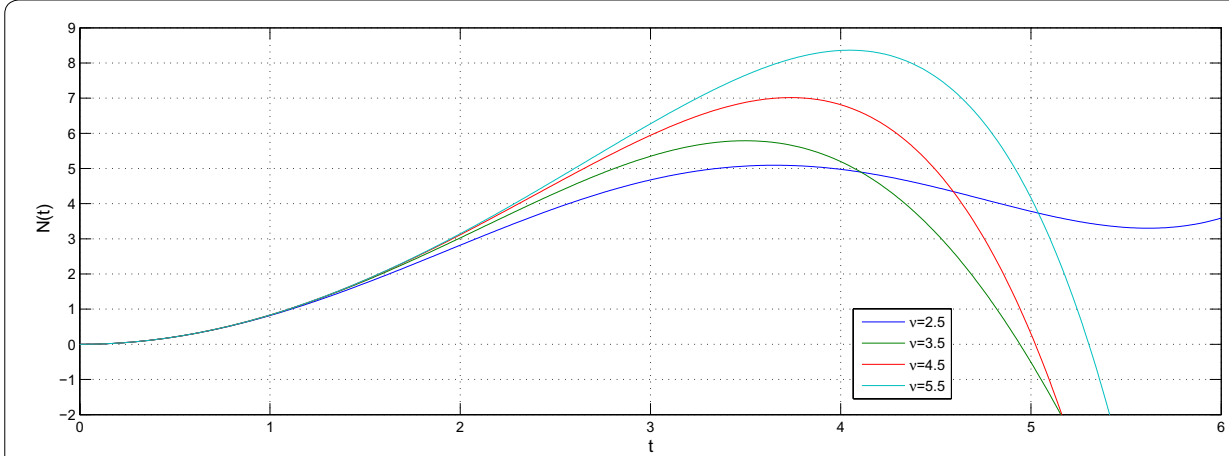

Fig. 5 Solution (38) for $a=1.5, N_{0}=1, \alpha=\mu=\xi=1$ and $b=c=l=e=1$

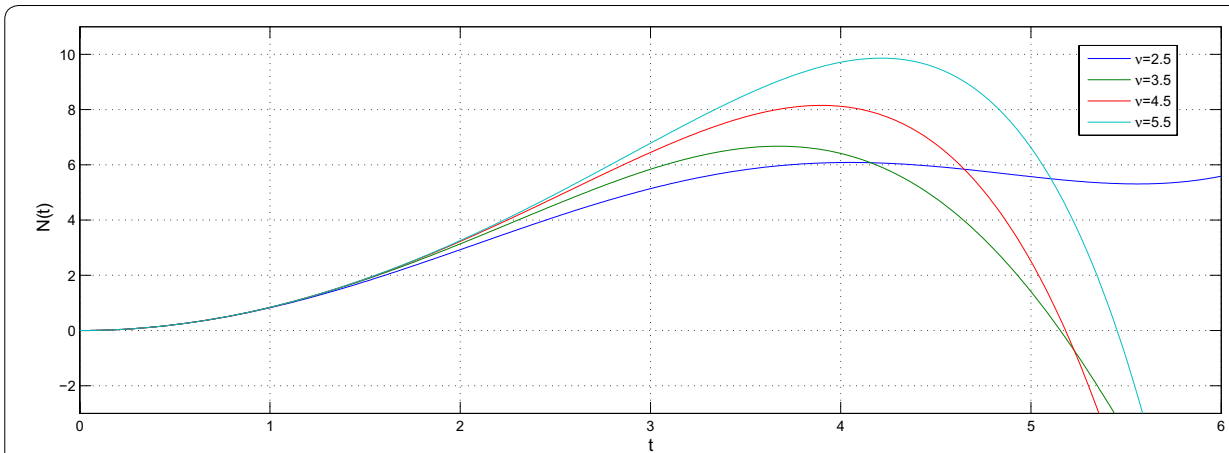

Fig. 6 Solution (40) for $a=2, N_{0}=1, \alpha=\mu=\xi=1$ and $b=c=l=e=1$

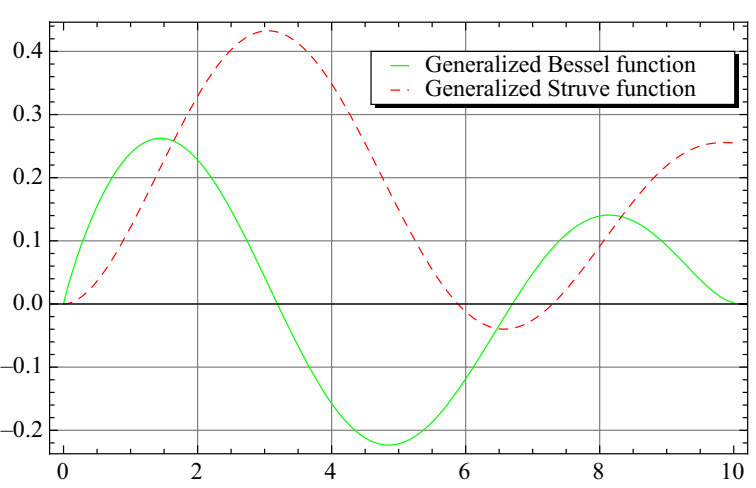

Fig. 7 Comparison between the Solution (38) for $v=\frac{1}{2}, a=1, N_{0}=1, \alpha=\mu=\xi=1$ and $b=c=l=e=1$ and (18) of Kumar et al. (2015)

\section{Conclusion}

In this paper, we investigated the integral transforms of Galue type generalization of Struve function and the results expressed in terms of Fox-Wright function. By substituting the appropriate value for the parameters, we obtained some results existing in the literature as corollaries. The results derived in section "Application" of this paper are general in character and likely to find certain applications in the theory of fractional 
calculus and special functions. The solutions of certain general families of fractional kinetic equations involving generalized GTSF presented in section "Conclusion". The main results given in section "Solution of fractional kinetic equation involving (11)" are general enough to be specialized to yield many new and known solutions of the corresponding generalized fractional kinetic equations. For instance, if we put $a=\alpha=\xi=1$ and $\mu=\frac{3}{2}$ in (34), (39) and (41), then we get the Eqs. (15), (19) and (24) of Nisar et al. (2016b).

\section{Authors' contributions}

All authors carried out the proofs of the main results. All authors read and approved the final manuscript.

\section{Author details}

Department of Mathematics, College of Arts and Science, Prince Sattam Bin Abdulaziz University, 11991 Wadi Aldawser, Saudi Arabia. ${ }^{2}$ Department of Mathematics and Computer Sciences, Faculty of Arts and Sciences, Çankaya University, 0630 Ankara, Turkey. ${ }^{3}$ Department of Mathematics, King Saud University, 12372 Riyadh, Saudi Arabia.

\section{Acknowledgements}

The research is supported by a grant from the "Research Center of the Center for Female Scientific and Medical Colleges", Deanship of Scientific Research, King Saud University. The authors are also thankful to visiting professor program at King Saud University for support.

\section{Competing interests}

The authors declare that they have no competing interests.

Received: 15 April 2016 Accepted: 10 June 2016

Published online: 29 June 2016

\section{References}

Adjabi Y, Jarad F, Baleanu D et al (2016) On Cauchy problems with Caputo Hadamard fractional derivatives. J Comput Anal Appl 21(4):661-681

Baleanu D, Moghaddam M, Mohammadi H et al (2016) A fractional derivative inclusion problem via an integral boundary condition. J Comput Anal Appl 21(3):504-514

Baricz Á (2010) Generalized Bessel functions of the first kind. Lecture Notes in Mathematics 1994. Springer, Berlin Bhowmick KN (1962) Some relations between a generalized Struve's function and hypergeometric functions. Vijnana Parishad Anusandhan Patrika 5:93-99

Bhowmick KN (1963) A generalized Struve's function and its recurrence formula. Vijnana Parishad Anusandhan Patrika $6: 1-11$

Chaurasia VBL, Kumar D (2010) On the solutions of generalized fractional kinetic equations. Adv Stud Theor Phy 4:773-780

Chaurasia VBL, Pandey SC (2008) On the new computable solution of the generalized fractional kinetic equations involving the generalized function for the fractional calculus and related functions. Astrophys Space Sci 317:213-219

Choi J, Kumar D (2015) Solutions of generalized fractional kinetic equations involving Aleph functions. Math Commun 20:113-123

Chouhan A, Purohit SD, Saraswat S (2013) An alternative method for solving generalized differential equations of fractional order. Kragujevac J Math 37:299-306

Chouhan A, Sarswat S (2012) On solution of generalized Kinetic equation of fractional order. Int J Math Sci Appl 2:813-818

Erdélyi A, Magnus W, Oberhettinger F, Tricomi FG (1954) Tables of integral transforms. McGraw-Hill, New York

Galué L (2003) A generalized Bessel function. Int Transforms Spec Funct 14:395-401

Glöckle WG, Nonnenmacher TF (1991) Fractional integral operators and Fox functions in the theory of viscoelasticity. Macromol Am Chem Soc 24:6426-6434

Gupta A, Parihar CL (2014) On solutions of generalized kinetic equations of fractional order. Bol Soc Paran Mat 32:181-189

Gupta VG, Sharma B (2011) On the solutions of generalized fractional kinetic equations. Appl Math Sci 5:899-910

Haubold HJ, Mathai AM (2000) The fractional kinetic equation and thermonuclear functions. Astrophys Space Sci 327:53-63

Hilfer R (2000) Applications of fractional calculus in physics. World Scientific, Singapore

Kanth BN (1981) Integrals involving generalized Struve's function. Nepali Math Sci Rep 6:61-64

Kilbas AA, Srivastava HM, Trujillo JJ (2006) Theory and applications of fractional differential equations. North-Holland Mathematics Studies 204, Elsevier, Amsterdam

Kilbas AA, Saigo M, Trujillo JJ (2002) On the generalized Wright function. Fract Calc Appl Anal 5:437-460

Kilbas AA, Sebastian N (2008) Generalized fractional integration of Bessel function of the first kind. Int Transf Spec Funct 19:869-883

Kilbas AA, Sebastian N (2010) Fractional integration of the product of Bessel function of the first kind. Fract Calc Appl Anal 13:159-175

Kiryakova V (1977) All the special functions are fractional differintegrals of elementary functions. J Phys A 30:5085-5103 
Kumar D, Purohit SD, Secer A, Atangana A (2015) On generalized fractional kinetic equations involving generalized Bessel function of the first kind. Math Probl Eng 2015:289387. doi:10.1155/2015/289387

Mathai AM, Saxena RK, Haubold HJ (2010) The H-Function: theory and applications. Springer, New York

Miller KS, Ross B (1993) An introduction to the fractional calculus and fractional differential equations. Wiley, New York Mittag-Leffler GM (1905) Sur la representation analytiqie d'une fonction monogene cinquieme note. Acta Math 29:101-181

Nisar KS, Atangana A (2016) Exact solution of fractional kinetic equation in terms of Struve functions. (Submitted)

Nisar KS, Agarwal P, Mondal SR (2016a) On fractional integration of generalized Struve functions of first kind. Adv Stud Contemp Math 26(1):63-70

Nisar KS, Purohit SD, Mondal SR (2016b) Generalized fractional kinetic equations involving generalized Struve function of the first kind. J King Saud Univ Sci 28(2):167-171. doi:10.1016/j.jksus.2015.08.005

Orhan H, Yagmur N (2013) Starlikeness and convexity of generalized Struve functions. Abstr Appl Anal. Art. ID 954513:6

Orhan H, Yagmur N (2014) Geometric properties of generalized Struve functions. Ann Alexandru loan Cuza Univ-Math. doi:10.2478/aicu-2014-0007

Podlubny I (1999) Fractional differential equations. Academic Press, New York

Rainville ED (1960) Special functions. Macmillan, New York

Saichev A, Zaslavsky M (1997) Fractional kinetic equations: solutions and applications. Chaos 7:753-764

Saigo M (1977) A remark on integral operators involving the Gauss hypergeometric functions. Math Rep Kyushu Univ 11:135-143

Samko SG, Kilbas AA, Marichev Ol, (1993) Fractional integrals and derivatives. Translated from the 1987 Russian original. Gordon and Breach, Yverdon

Saxena RK, Mathai AM, Haubold HJ (2002) On fractional kinetic equations. Astrophys Space Sci 282:281-287

Saxena RK, Mathai AM, Haubold HJ (2004) On generalized fractional kinetic equations. Phys A 344:657-664

Saxena RK, Mathai AM, Haubold HJ (2006) Solution of generalized fractional reaction-diffusion equations. Astrophys Space Sci 305:305-313

Saxena RK, Kalla SL (2008) On the solutions of certain fractional kinetic equations. Appl Math Comput 199:04-511

Singh RP (1974) Generalized Struve's function and its recurrence relations. Ranchi Univ Math J 5:67-75

Singh RP (1985) Generalized Struve's function and its recurrence equation. Vijnana Parishad Anusandhan Patrika 28:287-292

Singh RP (1988) Some integral representation of generalized Struve's function. Math Ed (Siwan) 22:91-94

Singh RP (1988) On definite integrals involving generalized Struve's function. Math Ed (Siwan) 22:62-66

Singh RP (1989) Infinite integrals involving generalized Struve function. Math Ed (Siwan) 23:30-36

Srivastava HM, Karlsson PW (1985) Multiple Gaussian Hypergeometric Series. Halsted Press (Ellis Horwood Limited, Chichester), Wiley, New York, Chichester, Brisbane and Toronto

Srivastava HM, Lin S-D, Wang P-Y (2006) Some fractional-calculus results for the $\mathrm{H}$-function associated with a class of Feynman integrals. Russ J Math Phys 13:94-100

Srivastava HM (2007) Some Fox-Wright generalized hypergeometric functions and associated families of convolution operators. Appl Anal Discr Math 1:56-71

Srivastava HM, Choi J (2012) Zeta and q-Zeta functions and associated series and integrals. Elsevier Science Publishers, Amsterdam

Srivastava HM, Saxena RK (2001) Operators of fractional integration and their applications. Appl Math Comput 118:1-52

Wiman A (1905) Uber den fundamental satz in der theorie der funktionen $E_{\alpha}(z)$. Acta Math 29:191-201

Wright EM (1940) The asymptotic expansion of integral functions defined by Taylor series. Philos Trans R Soc Lond Ser A 238:423-451

Wright EM (1940) The asymptotic expansion of the generalized hypergeometric function. Proc Lond Math Soc 46:389-408

Zaslavsky GM (1994) Fractional kinetic equation for Hamiltonian chaos. Phys D 76:110-122

\section{Submit your manuscript to a SpringerOpen ${ }^{\circ}$ journal and benefit from:}

- Convenient online submission

- Rigorous peer review

- Immediate publication on acceptance

Open access: articles freely available online

High visibility within the field

- Retaining the copyright to your article

Submit your next manuscript at $>$ springeropen.com 